\title{
A Basic Inequality for the Tanaka-Webster Connection
}

\author{
Dae Ho Jin ${ }^{1}$ and Jae Won Lee ${ }^{2}$ \\ ${ }^{1}$ Department of Mathematics, Dongguk University, Gyeongju 780-714, Republic of Korea \\ ${ }^{2}$ Department of Mathematics, Sogang University, Seoul 121-742, Republic of Korea \\ Correspondence should be addressed to Jae Won Lee, leejaewon@sogang.ac.kr
}

Received 7 November 2011; Accepted 28 November 2011

Academic Editor: C. Conca

Copyright (c) 2012 D. H. Jin and J. W. Lee. This is an open access article distributed under the Creative Commons Attribution License, which permits unrestricted use, distribution, and reproduction in any medium, provided the original work is properly cited.

For submanifolds tangent to the structure vector field in Sasakian space forms, we establish a Chen's basic inequality between the main intrinsic invariants of the submanifold (namely, its pseudosectional curvature and pseudosectional curvature on one side) and the main extrinsic invariant (namely, squared pseudomean curvature on the other side) with respect to the TanakaWebster connection. Moreover, involving the pseudo-Ricci curvature and the squared pseudomean curvature, we obtain a basic inequality for submanifolds of a Sasakian space form tangent to the structure vector field in terms of the Tanaka-Webster connection.

\section{Introduction}

One of the basic interests in the submanifold theory is to establish simple relationship between intrinsic invariants and extrinsic invariants of a submanifold. Gauss-Bonnet Theorem, Isoperimetric inequality, and Chern-Lashof Theorem are those such kind of study.

Chen [1] established a nice basic inequality-related intrinsic quantities and extrinsic ones of submanifolds in a space form with arbitrary codimension. Moreover, he studied the basic inequalities of submanifolds of complex space forms and characterize submanifolds when the equality holds.

In this paper, we introduce pseudosectional curvatures and pseudo-Ricci curvature for the Tanaka-Webster connection in a Sasakian space form. After then, we study basic inequalities for submanifolds of a Sasakian space form of a constant pseudosectional curvature and a pseudo-Ricci curvature in terms of the Tanaka-Webster connection. 


\section{Preliminaries}

Let $\widetilde{M}$ be an odd-dimensional Riemannian manifold with a Riemannian metric $\widetilde{g}$ satisfying

$$
\begin{gathered}
\eta(\xi)=1, \quad \varphi^{2}=-I+\eta \otimes \xi, \quad \eta(X)=\tilde{g}(X, \xi), \\
\tilde{g}(\varphi X, \varphi Y)=\tilde{g}(X, Y)-\eta(X) \eta(Y) .
\end{gathered}
$$

Then $(\varphi, \xi, \eta, \widetilde{g})$ is called the almost contact metric structure on $\widetilde{M}$. Let $\Phi$ denote the fundamental 2 form in $\widetilde{M}$ given by $\Phi(X, Y)=\widetilde{g}(X, \varphi Y)$ for all $X, Y \in T \widetilde{M}$, the set of vector fields of $\widetilde{M}$. If $\Phi=d \eta$, then $\widetilde{M}$ is said to be a contact metric manifold. Moreover, if $\xi$ is a Killing vector field with respect to $\tilde{g}$, and the contact metric structure is called a $\mathcal{K}$-contact structure. Recall that a contact metric manifold is $\mathcal{K}$-contact if and only if

$$
\tilde{\nabla}_{X} \xi=-\varphi X
$$

for any $X \in T \widetilde{M}$, where $\widetilde{\nabla}$ is the Levi-Civita connection of $\widetilde{M}$. The structure of $\widetilde{M}$ is said to be normal if $[\varphi, \varphi]+2 d \eta \otimes \xi-0$, where $[\varphi, \varphi]$ is the Nijenhuis torsion of $\varphi$. A Sasakian manifold is a normal contact metric manifold. In fact, an almost contact metric structure is Sasakian if and only if

$$
\left(\tilde{\nabla}_{X} \varphi\right) Y=\bar{g}(X, Y) \xi-\eta(Y) X
$$

for all vector fields $X$ and $Y$. Every Sasakian manifold is a $\mathcal{K}$-contact manifold.

Given a Sasakian manifold $\widetilde{M}$, a plane section $\pi$ in $T_{p} \widetilde{M}$ is called a $\varphi$-section if it spanned by $X$ and $\varphi X$, where $X$ is a unit tangent vector field orthogonal to $\xi$. The sectional curvature $\widetilde{K}(\pi)$ of a $\varphi$-section $\pi$ is called $\varphi$-sectional curvature. If a Sasakian manifold $\widetilde{M}$ has constant $\varphi$-sectional curvature $c, \widetilde{M}$ is called a Sasakian space form, denoted by $\widetilde{M}(c)$. (For more details, see [2]).

Now let $M$ be a submanifold immersed in $(\widetilde{M}, \varphi, \xi, \eta, g)$. We also denote by $g$ the induced metric on $M$. Let $T M$ be the Lie algebra of vector fields in $M$ and $T^{\perp} M$ the set of all vector fields normal to $M$. We denote by $h$ the second fundamental form of $M$ and by $A_{v}$ the Weingarten endomorphism associated with any $v \in T^{\perp} M$. We put $h_{i j}^{r}=\bar{g}\left(h\left(e_{i}, e_{j}\right), e_{r}\right)$ for any orthonormal vector $e_{i}, e_{j} \in T M$ and $e_{r} \in T^{\perp} M$. The mean curvature vector field $H$ is defined by $H=(1 / \operatorname{dim} M) \operatorname{trace}(h) . M$ is said to be totally geodesic if the second fundamental form vanishes identically.

From now on, we assume that the dimension of $M$ is $n+1$, and that of the ambient manifold $\widetilde{M}$ is $2 m+1(m \geq 2)$. We also assume that the structure vector field $\xi$ is tangent to $M$. Hence, if we denote by $D$ the orthogonal distribution to $\xi$ in $T M$, we have the orthogonal direct decomposition of $T M$ by $T M=D \oplus \operatorname{span}\{\xi\}$. For any $X \in T M$, we write $\varphi X=T X+$ $N X$, where $T X(N X$, resp.) is the tangential (normal, resp.) component of $\varphi X$. If $\varphi M$ is a $\mathcal{K}$-contact manifold, (2.2) gives

$$
h(X, \xi)=-N X,
$$


for any $X$ in $T M$. Given a local orthonormal frame $\left\{e_{1}, \ldots, e_{n}\right\}$ of $D$, we can define the squared norms of $T$ and $N$ by

$$
\|T\|^{2}=\sum_{i, j=1}^{n} g\left(e_{i}, T e_{j}\right)^{2}, \quad\|N\|^{2}=\sum_{i, j=1}^{n} g\left(e_{i}, N e_{j}\right)^{2},
$$

resepectively. It is easy to show that both $\|T\|^{2}$ and $\|N\|^{2}$ are independent of the choice of the orthonormal frames. The submanifold $M$ is said to be invariant if $N$ is identically zero, that is, $\varphi X \in T M$ for any $X \in T M$. On the other hand, $M$ is said to be an anti-invariant submanifold if $T$ is identically zero, that is, $\varphi X \in T^{\perp} M$ for any $X \in T M$.

\section{The Tanaka-Webster Connection for Sasakian Space Form}

The Tanaka-Webster connection $[3,4]$ is the canonical affine connection defined on a nondegenerate pseudo-Hermitian CR-manifold. Tanno [5] defined the Tanaka-Webster connection for contact metric manifolds by the canonical connection which coincides with the Tanaka-Webester connection if the associated CR-structure is integrable. We define the Tanaka-Webster connection for submanifolds of Sasakian manifolds by the naturally extended affine connection of Tanno's Tanaka-Webster connection. Now we recall the TanakaWebster connection $\hat{\nabla}$ for contact metric manifolds

$$
\hat{\nabla}_{X} Y=\tilde{\nabla}_{X} Y+\eta(X) \varphi Y+\left(\nabla_{X} \eta\right)(Y) \xi-\eta(Y) \nabla_{X} \xi
$$

for all vector fields $X, Y \in T \widetilde{M}$. Together with (2.1), $\widehat{\nabla}$ is written by

$$
\widehat{\nabla}_{X} Y=\tilde{\nabla}_{X} Y+\eta(X) \varphi Y+\eta(Y) \varphi X-\tilde{g}(Y, \varphi(X)) \xi .
$$

Also, by using (2.1) and (2.3), we can see that

$$
\widehat{\nabla} \eta=0, \quad \hat{\nabla} \xi=0, \quad \hat{\nabla} \varphi=0, \quad \widehat{\nabla} \tilde{g}=0 .
$$

We define the Tanaka-Webster curvature tensor of $\widetilde{R}$ (in terms of $\tilde{\nabla}$ ) by

$$
\widehat{R}(X, Y) Z=\hat{\nabla}_{X} \hat{\nabla}_{Y} Z-\hat{\nabla}_{Y} \hat{\nabla}_{Y} Z-\hat{\nabla}_{[X, Y]} Z,
$$

for all vector fields $X, Y$, and $Z$ in $\widetilde{M}$.

Let $\widetilde{M}(c)$ be a Sasakian space form of constant sectional curvature $c$ and $M$ a submanifold of $\widetilde{M}(c)$. Then, we have the following Gauss' equation:

$$
\begin{aligned}
\widehat{R}(X, Y) Z= & \frac{c+3}{4}[\{g(Y, Z)-\eta(Y) \eta(Z)\} X-\{g(X, Z)-\eta(X) \eta(Z)\} Y \\
& +\{g(X, Z) \eta(Y)-g(Y, Z) \eta(X)\} \xi+2 g(X, \varphi Y) \varphi Z] \\
+ & \frac{c+7}{4}\{g(Z, \varphi Y) \varphi X-g(Z, \varphi X) \varphi Y\}
\end{aligned}
$$

for any tangent vector fields $X, Y, Z$ tangent to $M$. 
Let us define the connection $\stackrel{\circ}{\nabla}$ on $M$ induced from the Tanaka-Webster connection $\hat{\nabla}$ on $\widetilde{M}$ given by

$$
\stackrel{\circ}{\nabla}_{X} Y=\stackrel{\circ}{\nabla}_{X} Y+\widehat{h}(X, Y)
$$

for any $X, Y \in \Gamma(T M)$, where $\widehat{h}$ is called the lightlike second fundamental form of $M$ with respect to the induced connection $\stackrel{\circ}{\nabla}$. In the view of (3.2) and (3.6),

$$
\stackrel{\circ}{\nabla}_{X} Y+\widehat{h}(X, Y)=\nabla_{X} Y+h(X, Y)+\eta(X) \varphi Y+\eta(Y) \varphi X-\bar{g}(Y, \varphi X) \xi
$$

From (3.7), we obtain

$$
\begin{gathered}
\stackrel{\circ}{\nabla}_{X} Y=\nabla_{X} Y+\eta(X) T Y+\eta(Y) T X-\bar{g}(Y, \varphi X) \xi, \\
\widehat{h}(X, Y)=h(X, Y)+\eta(X) N Y+\eta(Y) N X,
\end{gathered}
$$

where $\varphi X=T X+N X$.

From (3.3), (3.8), and (3.9) it is easy to verify the following:

$$
\stackrel{\circ}{\nabla} \eta=0, \quad \stackrel{\circ}{\nabla} \xi=0, \quad \stackrel{\circ}{\nabla} \varphi=0, \quad \stackrel{\circ}{\nabla} g=0 .
$$

Moreover, for the induced connection $\nabla$, we have the following

$$
\nabla_{X} \xi=-T X, \quad h(X, \xi)=-N X .
$$

From the definition of $\widehat{R}$, together with (3.5), we have

$$
\begin{aligned}
g(\stackrel{\circ}{R}(X, Y) Z, W)= & \frac{c+3}{4}[\{g(Y, Z)-\eta(Y) \eta(Z)\} g(X, W)-\{g(X, Z)-\eta(X) \eta(Z)\} g(Y, W) \\
& +\{g(X, Z) \eta(Y)-g(Y, Z) \eta(X)\} g(\xi, W)+2 g(X, \varphi Y) g(\varphi Z, W)] \\
& +\frac{c+7}{4}\{g(Z, \varphi Y) g(\varphi X, W)-g(Z, \varphi X) g(\varphi Y, W)\} \\
& +\bar{g}(\widehat{h}(X, W), \widehat{h}(Y, Z))-\bar{g}(\widehat{h}(X, Z), \widehat{h}(Y, W)),
\end{aligned}
$$

for any $X, Y, Z, W \in T M$.

For an orthonormal basis $\left\{e_{1}, \ldots, e_{n+1}\right\}$ of the tangent space $T_{p} M, p \in M$, the pseudoscalar curvature $\widehat{\tau}$ at $p$ is defined by

$$
\widehat{\tau}=\sum_{i<j} \widehat{K}\left(e_{i} \wedge e_{j}\right)
$$


where $\widehat{K}\left(e_{i} \wedge e_{j}\right)$ denotes the pseudosectional curvature of $M$ associated with the plane section spanned by $e_{i}$ and $e_{j}$ for the Tanaka-Webster connection $\hat{\nabla}$. In particular, if we put $e_{n+1}=\xi_{p}$, then (3.13) implies that

$$
2 \widehat{\tau}=\sum_{i \neq j} \widehat{K}\left(e_{i} \wedge e_{j}\right)+2 \sum_{i=1}^{n} \widehat{K}\left(e_{i} \wedge \xi\right)
$$

Moreover, from (3.9), we have

$$
\begin{gathered}
\widehat{h}_{i j}^{r}=h_{i j}, \quad i, j \in\{1, \ldots, n\}, \\
\widehat{h}_{i n+1}^{r}=0, \quad j \in\{1, \ldots, n+1\} .
\end{gathered}
$$

The pseudomean curvature vector field $H$ is defined by $\widehat{H}=(1 / \operatorname{dim} M) \operatorname{trace}(\widehat{h}) . M$ is said to be totally pseudogeodesic if the second fundamental $\widehat{h}$ form vanishes identically. From (2.5), (3.12) and (3.14), we obtain the following relationship between the pseudoscalar curvature and the pseudomean curvature of $M$,

$$
2 \widehat{\tau}=(n+1)^{2}\|\widehat{H}\|^{2}-\|\widehat{h}\|^{2}+n(n-1) \frac{c+3}{4}+\frac{3 c+13}{4}\|T\|^{2} .
$$

We now recall the Chen's lemma.

Lemma 3.1 (see [6]). Let $a_{1}, \ldots, a_{n}, c$ be $n+1(n \geq 2)$ real numbers such that

$$
\left(\sum_{i=1}^{n} a_{i}\right)^{2}=(n-1)\left(\sum_{i=1}^{n} a_{i}^{2}+c\right) .
$$

Then, $2 a_{1} a_{2} \geq c$, with the equality holding if and only if $a_{1}+a_{2}=a_{3}=\cdots=a_{n}$.

Let $p \in M$ and let $\pi$ be a plane section of $T_{p} M$ which is generated by orthonormal vectors $X$ and $Y$. We can define a function $\alpha(\pi)$ of tangent space $T_{p} M$ into [0,1] by

$$
\alpha(\pi)=g(T X, Y)^{2},
$$

which is well defined.

Now, we prove the following.

Theorem 3.2. Let $M$ be an $(n+1)$-dimensional $(n \geq 2)$ submanifold isometrically immersed in a $m$ dimensional Sasakian space form $\widetilde{M}(c)$ such that the structure vector field $\xi$ is tangent to $M$ in terms 
of the Tanaka-Wester connection $\hat{\nabla}$. Then, for each point $p \in M$ and each plane section $\pi \subset T_{p} M$, we have the following:

$$
\begin{aligned}
\widehat{\tau}-\widehat{K}(\pi) \leq & \frac{(n+1)^{2}(n-1)}{2 n}\|\widehat{H}\|^{2}+\frac{1}{8}(n+1)(n+2)(c+3) \\
& +\frac{3 c+13}{8}\|T\|^{2}-\frac{3 c+13}{4} \alpha(\pi) .
\end{aligned}
$$

Equality in (3.19) holds at $p \in M$ if and only if there exist an orthonormal basis $\left\{e_{1}, \ldots, e_{n+1}\right\}$ of $T_{p} M$ and an orthonormal basis $\left\{e_{n+2}, \ldots, e_{m}\right\}$ of $T_{p}^{\perp} M$ such that (a) $\pi=\operatorname{Span}\left\{e_{1}, e_{2}\right\}$ and (b) the shape operators $A_{r}=A_{e_{r}}, r=n+2, \ldots, m$, take the following forms:

$$
\begin{aligned}
A & =\left(\begin{array}{ccc}
a & 0 & 0 \\
0 & -a & 0 \\
0 & 0 & 0_{n-1}
\end{array}\right), \\
A & =\left(\begin{array}{ccc}
h_{11}^{r} & h_{12}^{r} & 0 \\
h_{12}^{r} & -h_{11}^{r} & 0 \\
0 & 0 & 0_{n-1}
\end{array}\right), \quad r=n+3, \ldots, m .
\end{aligned}
$$

Proof. Let $M^{n+1}$ be a submanifold of $\widetilde{M}(c)$. We introduce

$$
\widehat{\rho}=2 \widehat{\tau}-\frac{(n+1)^{2}(n-1)}{2 n}\|\widehat{H}\|^{2}-(n+1)(n+2) \frac{c+3}{4}-\frac{3 c+13}{4}\|T\|^{2} .
$$

Then, from (3.16) and (3.21), we get

$$
(n+1)\|\widehat{H}\|^{2}=n\|\widehat{h}\|^{2}+n\left(\widehat{\rho}-\frac{2(c+3)}{4}\right) .
$$

Let $p$ be a point of $M$ and let $\pi \subset T_{p} M$ be a plane section at $p$. We choose an orthonormal basis $\left\{e_{1}, \ldots, e_{n+1}\right\}$ for $T_{p} M$ and $\left\{e_{n+2}, \ldots, e_{m}\right\}$ for $T_{p}^{\perp} M$ such that $e_{n+1}=\xi, \pi=\operatorname{Span}\left\{e_{1}, e_{2}\right\}$, and the pseudomean curvature vector $\widehat{H}$ is parallel to $e_{n+2}$. Then, from (3.22), we get

$$
\left(\sum_{i=1}^{n+1} \widehat{h}_{i i}^{n+2}\right)^{2}=n\left(\sum_{i=1}^{n+1}\left(\widehat{h}_{i i}^{n+2}\right)^{2}+\sum_{i \neq j}\left(\widehat{h}_{i j}^{n+2}\right)^{2}+\sum_{r=n+3}^{m} \sum_{i, j}\left(\widehat{h}_{i j}^{r}\right)^{2}+\widehat{\rho}-\frac{2(c+3)}{4}\right)
$$

and so, by applying Lemma 3.1, we obtain

$$
2 \widehat{h}_{11}^{n+2} \widehat{h}_{22}^{n+2} \geq \sum_{i \neq j}\left(\widehat{h}_{i j}^{n+2}\right)^{2}+\sum_{r=n+3}^{m} \sum_{i, j}\left(\widehat{h}_{i j}^{r}\right)^{2}+\widehat{\rho}-\frac{2(c+3)}{4} .
$$


On the other hand, from (3.12), we have

$$
\widehat{K}(\pi)=\widehat{h}_{11}^{n+2} \widehat{h}_{22}^{n+2}-\left(\widehat{h}_{12}^{n+2}\right)^{2}+\sum_{r=n+3}^{m}\left(\widehat{h}_{11}^{r} \widehat{h}_{22}^{r}-\left(\widehat{h}_{12}^{r}\right)^{2}\right) \frac{c+3}{4}+\frac{3 c+13}{4} g^{2}\left(e_{1}, \varphi e_{2}\right) .
$$

Then, from (3.24) and (3.25), we get

$$
\begin{aligned}
\widehat{K}(\pi)= & \frac{\rho}{2}+\frac{3 c+13}{4} g^{2}\left(e_{1}, \varphi e_{2}\right)+\sum_{r=n+2}^{m} \sum_{j>2}\left(\left(\widehat{h}_{1 j}^{r}\right)^{2}+\left(\widehat{h}_{2 j}^{r}\right)^{2}\right)+\frac{1}{2} \sum_{i \neq j>2}\left(\widehat{h}_{i j}^{n+2}\right)^{2} \\
& +\frac{1}{2} \sum_{r=n+3}^{m} \sum_{i, j>2}\left(\widehat{h}_{i j}^{r}\right)^{2}+\frac{1}{2} \sum_{r=n+3}^{m}\left(\widehat{h}_{11}^{r}+\widehat{h}_{22}^{r}\right)^{2} \\
\geq & \frac{\rho}{2}+\frac{3 c+13}{4} \alpha(\pi) .
\end{aligned}
$$

Combining (3.21) and (3.27), the inequality (3.19) yields. If the equality in (3.19) holds, then the inequalities given by (3.24) and (3.27) become equalities. In this case, we have

$$
\begin{gathered}
\widehat{h}_{1 j}^{n+2}=\widehat{h}_{2 j}^{n+2}=\widehat{h}_{i j}^{n+2}=0, \quad i \neq j>2, \\
\widehat{h}_{1 j}^{r}=\widehat{h}_{2 j}^{r}=\widehat{h} i j^{r}=0, \quad r \in\{n+3, \ldots, m\} ; i, j \in\{3, \ldots, n+1\}, \\
\widehat{h}_{11}^{n+3}+\widehat{h}_{22}^{n+3}=\cdots=\widehat{h}_{11}^{m}+\widehat{h}_{22}^{m}=0 .
\end{gathered}
$$

Moreover, choosing $e_{1}$ and $e_{2}$ such that $h_{12}^{n+2}=0$, from (3.11), we also have the following

$$
\widehat{h}_{11}^{n+2}+\widehat{h}_{22}^{n+2}=\widehat{h}_{33}^{n+2}=\cdots=\widehat{h}_{n+1 n+1}^{n+2}=0 .
$$

Thus, with respect to the chosen orthonormal basis $\left\{e_{1}, \ldots, e_{m}\right\}$, the shape operators of $M$ take the forms.

We now define a well-defined function $\widehat{\delta}_{M}$ on $M$ by using $(\inf \widehat{K})(p)=\inf \{\widehat{K}(\pi) \mid$ $\pi$ is a plane section $\left.\subset T_{p} M\right\}$ in the following manner:

$$
\widehat{\delta}_{M}=\widehat{\tau}-\inf \widehat{K} .
$$

If $c=-13 / 3$, then we obtain directly from (3.19) the following result.

Corollary 3.3. Let $M$ be an $(n+1)$-dimensional $(n \geq 2)$ submanifold isometrically immersed in a $m$-dimensional Sasakian space form $\widetilde{M}(-13 / 3)$ such that the structure vector field $\xi$ is tangent to $M$ in terms of the Tanaka-Wester connection $\hat{\nabla}$. Then, for each point $p \in M$ and each plane section $\pi \subset T_{p} M$, we have the following:

$$
\widehat{\delta}_{M} \leq \frac{(n+1)^{2}(n-1)}{2 n}\|\widehat{H}\|^{2}-\frac{1}{6}(n+1)(n+2) .
$$

The equality in (3.31) holds if and only if $M$ is a anti-invariant submanifold with $\operatorname{rank}(T)=2$. 
Proof. In order to estimate $\widehat{\delta}_{M}$, we minimize $\|T\|^{2}-2 \alpha(\pi)$ in (3.19). For an orthonormal basis $\left\{e_{1}, \ldots, e_{n+1}\right\}$ of $T_{p} M$ with $\pi=\operatorname{Span}\left\{e_{1}, e_{2}\right\}$, we write

$$
\|T\|^{2}-2 \alpha(\pi)=\sum_{i, j=3}^{n+1} g^{2}\left(e_{i}, \varphi e_{j}\right)+2 \sum_{j=3}^{n+1}\left\{g^{2}\left(e_{1}, \varphi e_{j}\right)+g^{2}\left(e_{2}, \varphi e_{j}\right)\right\} .
$$

Thus, we see that the minimum value of $\|T\|^{2}-2 \alpha(\pi)$ is zero, provided that $\pi=\operatorname{Span}\left\{e_{1}, e_{2}\right\}$ is orthogonal to $\xi$, and $\operatorname{span}\left\{\varphi e_{j} \mid j=3, \ldots, n\right\}$ is orthogonal to $T_{p} M$. Thus we have (3.31) with equality case holding if and only if $M$ is anti-invariant such that $\operatorname{rank}(T)=2$.

\section{A Pseudo-Ricci Curvature for Sasakian Space Form}

We denote the set of unit vectors in $T_{p} M$ by $T_{p}^{1} M$ by

$$
T_{p}^{1} M=\left\{X \in T_{p} M \mid g(X, X)=1\right\}
$$

Let $\left\{e_{1}, \ldots, e_{k}\right\}, 2 \leq k \leq n$, be an orthonormal basis of a $k$-place section $\Pi_{k}$ of $T_{p} M$. If $k=n$, then $\Pi_{k}=T_{p} M$, and if $k=2$, then $\Pi_{2}$ is a plane section of $T_{p} M$. For a fixed $i \in\{1, \ldots, k\}$, a $k$-pseudo-Ricci curvature of $\Pi_{k}$ at $e_{i}$, denoted by $\widehat{\operatorname{Ric}}_{\Pi_{k}}\left(e_{i}\right)$, is defined by [7]

$$
\widehat{\operatorname{Ric}}_{\Pi_{k}}\left(e_{i}\right)=\sum_{j \neq i}^{k} \widehat{K}_{i j}
$$

where $\widehat{K}_{i j}$ is the pseudosectional curvature in terms of the Tanaka-Webster connection $\hat{\nabla}$ of the plane section spanned by $e_{i}$ and $e_{j}$. We note that an $n$-pseudo-Ricci curvature $\operatorname{Ric}_{T_{p} M}\left(e_{i}\right)$ is the usual pseudo-Ricci curvature of $e_{i}$, denoted by $\widehat{\operatorname{Ric}}\left(e_{i}\right)$. Thus, for any orthonormal basis $\left\{e_{1}, \ldots, e_{n+1}\right\}$ for $T_{p} M$ and for a fixed $i \in\{1, \ldots, n+1\}$, we have the following:

$$
\widehat{\operatorname{Ric}}_{T_{p} M}\left(e_{i}\right)=\widehat{\operatorname{Ric}}\left(e_{i}\right)=\sum_{j \neq i}^{n+1} \widehat{K}_{i j}
$$

The pseudoscalar curvature $\widehat{\tau}\left(\Pi_{k}\right)$ of the $k$-plane section $\Pi_{k}$ is given by

$$
\widehat{\tau}\left(\Pi_{k}\right)=\sum_{1 \leq i<j \leq n+1} \widehat{K}_{i j}
$$

The relative null spae of $M$ at $p$ is defined by [8]

$$
\mathcal{N}_{p}=\left\{X \in T_{p} M \mid \widehat{h}(X, Y)=0, \forall Y \in T_{p} M\right\}
$$


Theorem 4.1. Let $\widetilde{M}(c)$ be a m-dimensional Sasakian space form and $M$ an $n+1$-dimensional submanifold tangent to $\xi$ with respect to the Tanaka-Webster connection $\hat{\nabla}$. Then,

(i) for each unit vector $X \in T_{p} M$ orthogonal to $\xi$, we have

$$
4 \widehat{\operatorname{Ric}}(X) \leq(n+1)^{2}\|\widehat{H}\|^{2}+(n-1)(c+3)+(3 c+13)\|T X\|^{2},
$$

(ii) if $\widehat{H}(p)=0$, then a unit tanget vector $X \in T_{p} M$ orthogonal to $\xi$ satisfies the equality case of (4.6) if and only of $X \in \mathcal{N}_{p}$.

(iii) the equality case of (4.6) holds identically for all unit tangent vectors orthogonal to $\xi$ at $p$ if and only if $p$ is a totally pseudogeodesic point in terms of the Tanaka-Webster connection.

Proof. (i) Let $X \in T_{p} M$ be a unit tangent vector at $p$, orthogonal to $\xi$. We choose an orthonormal basis $\left\{e_{1}, \ldots, e_{n+1}\right\}$ for $T_{p} M$ and $\left\{e_{n+2}, \ldots, e_{m}\right\}$ for $T_{p}^{\perp} M$ such that $e_{1}=X$ and $e_{n+1}=\xi$. Then, from (3.16), we have

$$
(n+1)^{2}\|\widehat{H}\|^{2}=2 \widehat{\tau}+\|\widehat{h}\|^{2}-n(n-1) \frac{c+3}{4}-\frac{3 c+13}{4}\|T\|^{2} .
$$

From (4.7), we get

$$
\begin{aligned}
(n+1)^{2}\|\widehat{H}\|^{2}= & 2 \widehat{\tau}+\sum_{r=n+2}^{m}\left[\left(\widehat{h}_{11}^{2}\right)^{2}+\left(\widehat{h}_{22}^{r}+\cdots+\widehat{h}_{n+1 n+1}^{r}\right)^{2}+2 \sum_{i<j}\left(\widehat{h}_{i j}^{r}\right)^{2}\right] \\
& -2 \sum_{r=n+2}^{m} \sum_{2 \leq i<j \leq n} \widehat{h}_{i i}^{r} \widehat{h}_{j j}^{r}-n(n-1) \frac{c+3}{4}-\frac{3 c+13}{4}\|T\|^{2} \\
= & 2 \widehat{\tau}+\frac{1}{2} \sum_{r=n+2}^{m}\left[\left(\widehat{h}_{11}^{r}+\widehat{h}_{22}^{r}+\cdots+\widehat{h}_{n+1 n+1}^{r}\right)^{2}+\left(\widehat{h}_{11}^{r}-\widehat{h}_{22}^{r}-\cdots-\widehat{h}_{n+1 n+1}^{r}\right)^{2}\right] \\
& +2 \sum_{n+2}^{m} \sum_{i<j}\left(\widehat{h}_{i j}^{r}\right)^{2}-2 \sum_{r=n+2}^{m} \sum_{2 \leq i<j \leq n} \widehat{h}_{i i}^{r} \widehat{h}_{j j}^{r}-n(n-1) \frac{c+3}{4}-\frac{3 c+13}{4}\|T\|^{2} .
\end{aligned}
$$

From (3.12), we have

$$
\widehat{K}_{i j}=\sum_{r=n+2}^{m}\left[\widehat{h}_{i i}^{r} \widehat{h}_{j j}^{r}-\left(\widehat{h}_{i j}^{r}\right)^{2}\right]+\frac{c+3}{4}+\frac{3 c+13}{4} g^{2}\left(e_{i}, T e_{j}\right),
$$

and consequently

$$
\sum_{2 \leq i<j \leq n+1} \widehat{K}_{i j}=\sum_{r=n+2}^{m}\left[\widehat{h}_{i i}^{r} \widehat{h}_{j j}^{r}-\left(\widehat{h}_{i j}^{r}\right)^{2}\right]+\frac{(n-1)(n-2)(c+3)}{8}+\frac{3 c+13}{8}\left\{\|T\|^{2}-2\left\|T e_{1}\right\|^{2}\right\} .
$$


Substituting (4.10) into (4.8), one gets

$$
\begin{aligned}
(n+1)^{2}\|\widehat{H}\|^{2} \geq & 2 \widehat{\tau}+\frac{(n+1)^{2}}{2}\|\widehat{H}\|^{2}+2 \sum_{r=n+2}^{m} \sum_{j=2}\left(\widehat{h}_{1 j}^{r}\right)^{2}-2 \sum_{2 \leq i<j \leq n+1} \widehat{K}_{i j} \\
& -\frac{(n-1)(c+3)}{2}-\frac{3 c+13}{2}\left\|T e_{1}\right\|^{2} .
\end{aligned}
$$

Therefore,

$$
\frac{(n+1)^{2}}{2}\|\widehat{H}\|^{2} \geq 2 \widehat{\operatorname{Ric}}(X)-\frac{(n-1)(c+3)}{2}-\frac{3 c+13}{2}\|T X\|^{2},
$$

which is equivalent to (4.6)

(ii) Assume that $\widehat{H}(p)=0$. Equality holds in (4.6) if and only if

$$
\begin{gathered}
\widehat{h}_{12}^{r}=\cdots=\widehat{h}_{1 n+1}^{r}=0, \\
\widehat{h}_{11}^{r}=\widehat{h}_{22}^{r}+\cdots+\widehat{h}_{n+1 n+1}^{r}, \quad r \in\{n+2, \ldots, m\} .
\end{gathered}
$$

Then, $\widehat{h}_{1 j}^{r}=0$ for each $j \in\{1, \ldots, n+1\}, r \in\{n+2, \ldots, m\}$, that is, $X \in \mathcal{N}_{p}$.

(iii) The equality case of (4.6) holds for all unit tangent vectors at $p$ if and only if

$$
\begin{gathered}
\widehat{h}_{i j}^{r}=0, \quad i \neq j, r \in\{n+2, \ldots, m\}, \\
\widehat{h}_{11}^{r}+\cdots+\widehat{h}_{n+1 n+1}^{r}-2 \widehat{h}_{i i}^{r}=0, \quad i \in\{1, \ldots, n+1\}, r \in\{n+2, \ldots, m\} .
\end{gathered}
$$

Since $\widehat{h}\left(e_{i}, e_{n+1}=\xi\right)=0$ from (3.10), $p$ is a totally pseudogeodesic point, and, hence, $\varphi\left(T_{p} M\right) \subset$ $T_{p} M$. The converse is trivial.

Corollary 4.2. Let $M$ be an $n+1$-dimensional invariant submanifold of a Sasakian space form $\widetilde{M}(c)$. Then,

(i) for each unit vector $X \in T_{p} M$ orthogonal to $\xi$, we have

$$
4 \widehat{\operatorname{Ric}}(X) \leq(n-1)(c+3)+(3 c+13)
$$

(ii) A unit tanget vector $X \in T_{p} M$ orthogonal to $\xi$ satisfies the equality case of (4.6) if and only if $X \in \mathcal{N}_{p}$.

(iii) The equality case of (4.6) holds identically for all unit tangent vectors orthogonal to $\xi$ at $p$ if and only if $p$ is a totally pseudogeodesic point in terms of the Tanaka-Webster connection.

\section{References}

[1] B.-Y. Chen, "A Riemannian invariant for submanifolds in space forms and its applications," in Geometry and Topology of Submanifolds, pp. 58-81, World Scientific, River Edge, NJ, USA, 1994. 
[2] D. E. Blair, Riemannian Geometry of Contact and Symplectic Manifolds, vol. 203, Birkhäuser Boston, Boston, Mass, USA, 2002.

[3] N. Tanaka, "On non-degenerate real hypersurfaces, graded Lie algebras and Cartan connections," Japanese Journal of Mathematics, vol. 2, no. 1, pp. 131-190, 1976.

[4] S. M. Webster, "Pseudo-Hermitian structures on a real hypersurface," Journal of Differential Geometry, vol. 13, no. 1, pp. 25-41, 1978.

[5] S. Tanno, "Variational problems on contact Riemannian manifolds," Transactions of the American Mathematical Society, vol. 314, no. 1, pp. 349-379, 1989.

[6] B.-Y. Chen, "Some pinching and classification theorems for minimal submanifolds," Archiv der Mathematik, vol. 60, no. 6, pp. 568-578, 1993.

[7] B.-Y. Chen, "Mean curvature and shape operator of isometric immersions in real-space-forms," Glasgow Mathematical Journal, vol. 38, no. 1, pp. 87-97, 1996.

[8] B.-Y. Chen, "Relations between Ricci curvature and shape operator for submanifolds with arbitrary codimensions," Glasgow Mathematical Journal, vol. 41, no. 1, pp. 33-41, 1999. 


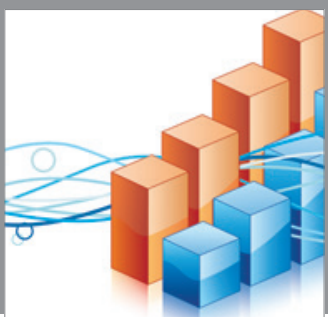

Advances in

Operations Research

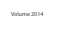

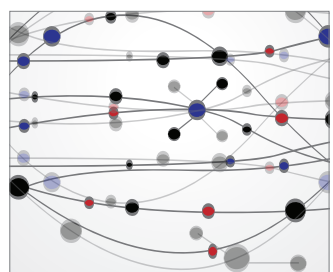

\section{The Scientific} World Journal
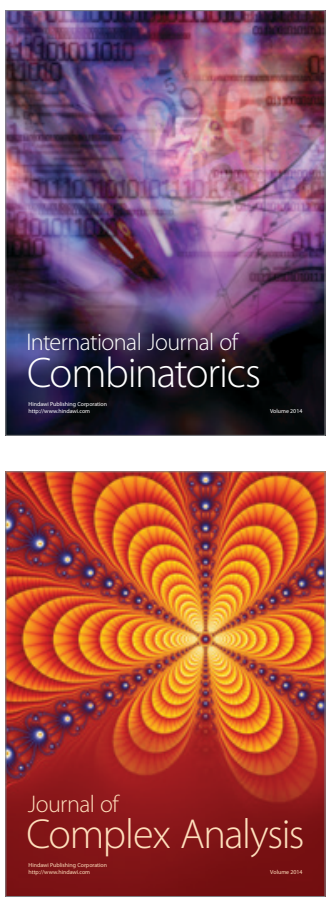

International Journal of

Mathematics and

Mathematical

Sciences
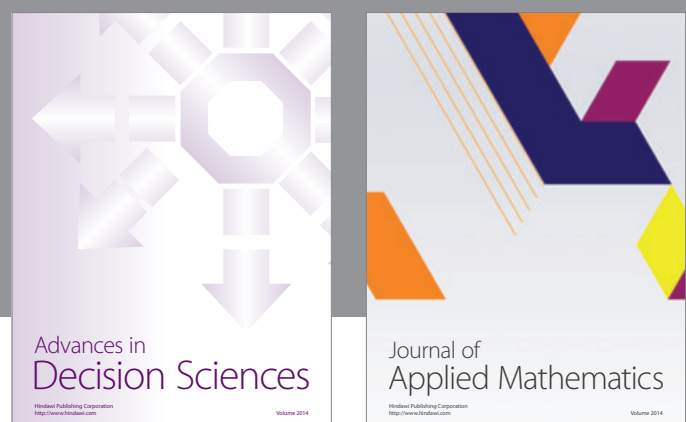

Journal of

Applied Mathematics
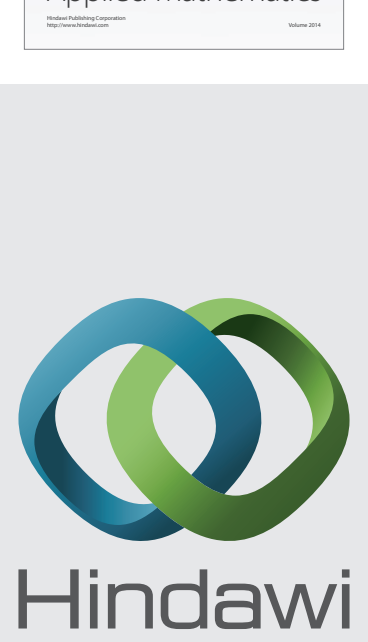

Submit your manuscripts at http://www.hindawi.com
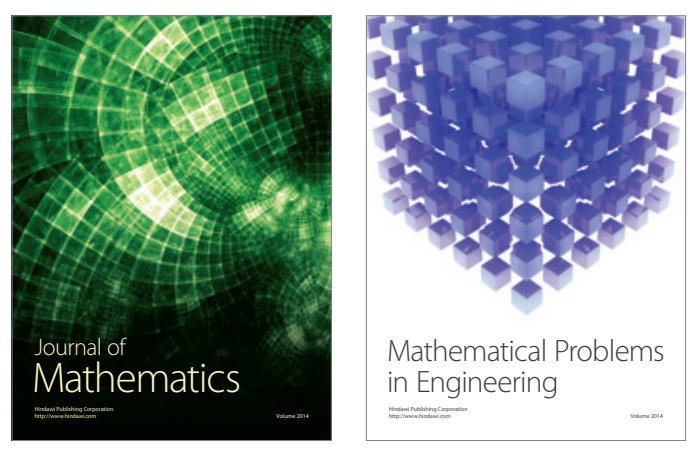

Mathematical Problems in Engineering
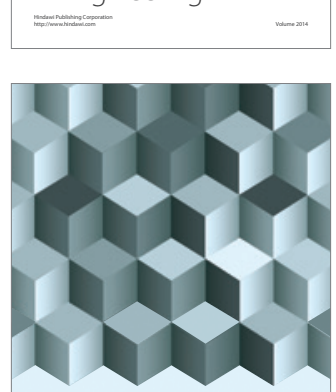

Journal of

Function Spaces
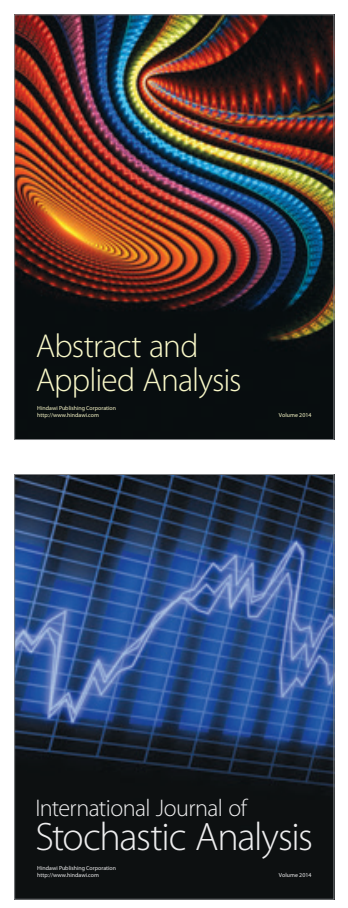

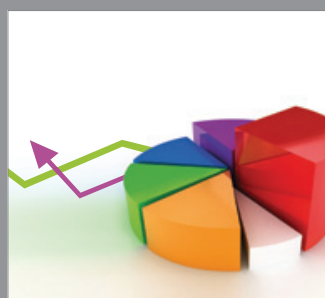

ournal of

Probability and Statistics

Promensencen
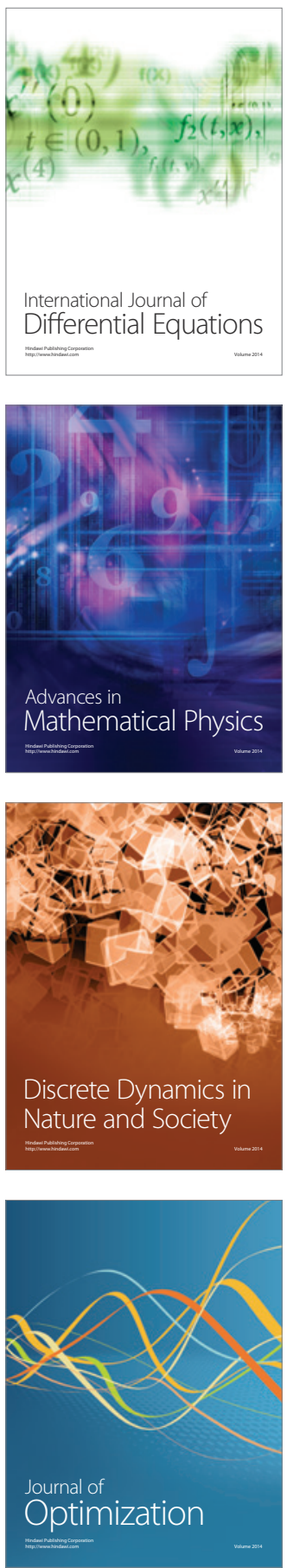